\title{
Comportamentos de coping no contexto da hospitalização infantil
}

\author{
Coping behaviors in the child \\ hospitalization context
}

\author{
Alessandra Brunoro MOTTA ${ }^{1}$ \\ Gimol Benzaquen PEROSA ${ }^{2}$ \\ Luisa BARROS ${ }^{3}$ \\ Kelly Ambrósio SILVEIRA ${ }^{4}$ \\ Ana Sofia da Silva LIMA $^{5}$ \\ Luciana Esgalha CARNIER ${ }^{6}$ \\ Paula Coimbra da Costa Pereira HOSTERT ${ }^{1}$ \\ Fernanda Rosalém CAPRINI ${ }^{7}$
}

\begin{abstract}
Resumo
A criança hospitalizada precisa lidar com estressores que geram ansiedade e sofrimento. Com o objetivo de descrever comportamentos de coping de crianças hospitalizadas e suas relações com idade, sexo e motivo da hospitalização, foram analisadas respostas ao Instrumento de Avaliação das Estratégias de Enfrentamento da Hospitalização de 148 crianças (6-12 anos, $M=9,5$ anos), a partir de um banco de dados integrado. Os resultados foram analisados por estatística descritiva e inferencial. Tomar remédio, conversar, assistir televisão, rezar e brincar foram os comportamentos mais referidos. Houve correlação entre comportamentos de coping e diferenças decorrentes do motivo da hospitalização. Meninas cantam mais e referiram mais choro, tristeza e medo do que meninos. Não foram verificadas diferenças em relação à idade, mas o comportamento de chantagem diminuiu em função da maior idade. Sugere-se que variáveis como sexo, motivo da hospitalização e idade sejam consideradas em intervenções com foco no coping da hospitalização.
\end{abstract}

Palavras-chave: Coping; Criança hospitalizada; Hospitalização.

$\nabla \nabla \nabla$

1 Universidade Federal do Espírito Santo, Departamento de Fonoaudiologia, Programa de Pós-Graduação em Psicologia. Av. Fernando Ferrari, 514, 29060-970, Vitória, ES, Brasil. Correspondência para/Correspondence to: A.B. MOTTA. E-mail: <alessandrabmotta@yahoo.com.br>.

2 Universidade Estadual Paulista Júlio de Mesquita Filho, Faculdade de Medicina, Departamento de Neurologia, Psicologia e Psiquiatria. Botucatu, SP, Brasil.

3 Universidade de Lisboa, Faculdade de Psicologia, Programa de Pós-Graduação em Psicologia da Saúde. Lisboa, Portugal.

${ }^{4}$ Psicóloga. Vitória, ES, Brasil.

5 Psicóloga. Lisboa, Portugal.

${ }^{6}$ Universidade Estadual Paulista Júlio de Mesquita Filho, Faculdade de Medicina, Hospital das Clínicas. Botucatu, SP, Brasil.

7 Centro Universitário São Camilo, Curso de Psicologia. Cachoeiro de Itapemirim, ES, Brasil.

Apoio: Conselho Nacional de Desenvolvimento Científico e Tecnológico (Processo no 485564/2006-8) e Ministério de Ciência e Tecnologia (Processo n 481483/2009-8), Coordenação de Aperfeiçoamento de Pessoal de Nível Superior e Fundação de Amparo à Pesquisa do Estado de São Paulo.

Agradecimento Especial à Profa. Dra. Sônia Regina Fiorim Enumo, que contribuiu com valiosas sugestões para a elaboração deste artigo. 


\begin{abstract}
Child hospitalization needs to cope with the stressors that cause anxiety and suffering. Aiming at describing the coping behaviors of hospitalized children and their relationships to age, sex and the reason for hospitalization, 148 responses of children aged 6-12 were analyzed ( $M=9.5$ years old) to the Coping with Hospitalization Scale, from an integrated database. The results were analyzed by descriptive and inferential statistics. Taking some medicine, talking, watching television, praying and playing were the most referred to behaviors. There was a correlation between the coping behaviors and the differences caused by the hospitalization reason. Girls tend to sing more, and demonstrated more crying, sorrow and fear than boys. There were no differences as to age, but persuasion behavior decreases in older children. We suggest that sex, hospitalization reason and age have to be considered in interventions on coping with hospitalization.
\end{abstract}

Keywords: Coping; Child hospitalized; Hospitalization.

Hospitalizações motivadas por condições crônicas e agudas de saúde são acompanhadas, em maior ou menor intensidade, pelo encontro da criança com uma situação nova, ameaçadora, em que há restrição do controle sobre os eventos. Representam, também, o afastamento de atividades do cotidiano infantil, tal como brincar e estudar e a exposição a procedimentos médicos invasivos, responsáveis por gerar medo, raiva, entre outras emoções com conotação negativa (Barros, 2003; Haiat, Bar-Mor, \& Shochat, 2003).

Quando a hospitalização se deve ao tratamento de doenças crônicas e de doenças que representam condições mais graves e ameaçadoras, como o câncer, há a incerteza quanto ao futuro e o temor de efeitos físicos do tratamento em curto e médio prazo, os quais podem gerar significativo estresse e sofrimento psicológico (Rodriguez et al., 2012). Compas, Jaser, Dunn e Rodriguez (2012) esclarecem que, tanto a criança, quanto os pais são, colocados diante do estresse agudo presente no momento do diagnóstico, bem como do estresse crônico, ao longo do tratamento. Em ambas as situações, agudas e crônicas, é preciso lidar com a falta de controlabilidade do estressor e com as restrições impostas pela rotina de tratamento e por características da própria doença.

Nesse contexto permeado de estressores, a criança pode utilizar estratégias para a sua autorregulação comportamental, de modo a alterar ou diminuir as fontes de estresse, colaborando para a adaptação frente às situações adversas. Tal processo tem sido referido como coping ou enfrentamento 332 (Compas, Connor-Smith, Saltzman, Thomsen, \&
Wadsworth, 2001). Analisado sob uma perspectiva desenvolvimentista, este constructo mostra-se de grande valia, dada a sua importância na relação entre eventos estressantes e resultados, tanto físicos quanto emocionais de curto e longo prazo (Compas \& Boyer, 2001).

De modo geral, as crianças empregam uma variedade de estratégias de enfrentamento para lidar com o estresse decorrente de cuidados médicos e de doença (Marsac, Donlon, Winston, \& Kassam-Adams, 2011). Analisando os resultados de estudos empíricos na literatura internacional sobre coping em crianças e adolescentes com doenças crônicas, Compas et al. (2012) verificaram relações entre as Estratégias de Enfrentamento (EE) e o ajustamento da criança e do adolescente: estratégias de controle secundário, que incluem pensamento positivo, aceitação e distração, por exemplo, se associaram a um melhor ajustamento em crianças e adolescentes; em oposição, estratégias de esquiva e negação se relacionaram com pior ajustamento.

No Brasil, estudos sobre coping infantil em contextos médicos ainda são escassos (Carnier, 2010; Ramos, 2012) e desafios teóricos e metodológicos descritos na literatura internacional (Compas et al., 2012; Skinner, Edge, Altman, \& Sherwood, 2003) têm guiado algumas propostas de avaliação. Um exemplo é o Instrumento de Avaliação do Enfrentamento da Hospitalização (AEH) (Motta \& Enumo, 2002; 2010), com aplicação em diferentes tipos de doença e/ou condição de hospitalização: câncer (Caprini, 2014; Lima, Barros, \& Enumo, 2014; Motta \& Enumo, 2010), anemia falciforme (Garioli, 2011; Oliveira, 2013), internação pré-cirúrgica (Carnier, 
2010), e internação em pediatria geral (Moraes \& Enumo, 2008).

No Instrumento de Avaliação do Enfrentamento da Hospitalização, adota-se um aporte teórico que considera o coping como regulação da ação em condição de estresse (Skinner \& Wellborn, 1994). Segundo esses autores, coping se refere ao "[...] modo como as pessoas regulam seu comportamento, emoção e orientação em condição de estresse psicológico" (p.112). Esta definição está fundamentada em um modelo motivacional, no qual os indivíduos buscam atender suas necessidades básicas de relacionamento, competência e autonomia que, em situação de estresse, encontram-se desafiadas ou ameaçadas.

Em termos metodológicos, o AEH considera a contribuição de Skinner et al. (2003), que propuseram uma estrutura hierárquica do constructo do coping. Esta estrutura incorpora, em sua base, as instâncias de enfrentamento (instances of coping), os comportamentos relatados ou observados diante do estressor, os quais podem ser organizados em categorias com definições claras e mutuamente exclusivas, nomeadas "estratégias de enfrentamento" (ways of coping), representando tipos de ações dirigidas ao manejo do estressor. Em um nível acima na estrutura do coping, estão categorias de ordem superior, denominadas "famílias de enfrentamento" (family of coping), que agregam estratégias de enfrentamento com semelhante função adaptativa. Por fim, alcançando o topo da estrutura, essas famílias de coping se relacionam com os processos adaptativos.

Ao integrar estudos sobre coping e regulação, é possível encontrar as bases desenvolvimentistas do coping (Zimmer-Gembeck \& Skinner, 2009). Segundo essas autoras, as ações reflexas dos bebês são substituídas por esquemas de ação coordenados, permitindo, por exemplo, que o bebê procure pelo cuidador ou por um brinquedo de sua preferência para se acalmar. Somente por volta de 2 anos é que a criança começa a apresentar as primeiras repostas voluntárias de coping, de modo que até os 5 anos é observado o manejo comportamental, tal como o uso da distração comportamental e do brincar de faz de conta para lidar com uma situação de sofrimento. Com o avanço cogni- tivo observado a partir de 6 anos, estratégias cognitivas podem ser adotadas, expressas em termos de distração cognitiva e resolução do problema, por exemplo. Na adolescência e no início da vida adulta, habilidades metacognitivas são somadas ao repertório existente, permitindo que metas em longo prazo e estratégias para evitar o estressor passem a ser observadas (Zimmer-Gembeck \& Skinner, 2009; 2011).

Os dados apresentados reforçam a necessidade de investigações com foco no enfrentamento da hospitalização entre as crianças, considerando fatores como a idade. As circunstâncias para a entrada no hospital (Franck et al., 2014) e o sexo da criança (Lynch, Kashikar-Zuck, Goldschneider, \& Jones, 2007; Vierhaus, Lohaus, \& Schmitz, 2011) são outros fatores em destaque neste estudo. No caso da hospitalização por câncer, o estudo adota um caráter intercultural, na medida em que analisou dados provenientes do Brasil e de Portugal.

Desse modo, o presente estudo teve como objetivo geral descrever e analisar os comportamentos de coping de crianças frente à hospitalização. Tendo em vista que tais comportamentos já foram analisados para o tratamento oncológico (Caprini, 2014; Hostert, 2010; Lima, 2009; Motta \& Enumo, 2010), para procedimentos cirúrgicos gerais (Carnier, 2010) e para o tratamento clínico de doenças diversas (Moraes \& Enumo, 2008), este estudo, especificamente, procurou responder às seguintes questões: (a) se há correlação entre comportamentos de coping mais referidos por crianças hospitalizadas; (b) se comportamentos de coping de crianças hospitalizadas diferem em função de características sociodemográficas, como idade e sexo; e (c) se comportamentos de coping de crianças hospitalizadas diferem em função do motivo da hospitalização (tipo de doença e/ou condição: câncer, cirurgia eletiva, doenças em geral). Ainda, a respeito do motivo da hospitalização, este estudo analisou diferenças entre crianças com câncer brasileiras e portuguesas.

\section{Método}

A partir da integração de dados de pesquisas realizadas com crianças hospitalizadas (Caprini, 
2014; Carnier, 2010; Hostert, 2010; Lima, 2009; Moraes, 2007; Motta, 2007), foi composta uma amostra de dados secundários, com 148 pacientes, sendo 61 internados para tratamento de câncer (41,2\%), 59 para cirurgia eletiva (39,9\%), e 28 para tratamento de doenças diversas $(18,2 \%)$. Essas crianças tinham, em média, 9,5 anos $( \pm 1,94$; variação $=6-13)$, sendo 61 meninas $(41,2 \%)$ e 87 meninos (58,8\%).

Entre as crianças hospitalizadas com câncer, 19 eram portuguesas e estavam em tratamento no Instituto Português de Oncologia de Lisboa Francisco Gentil, localizado em Lisboa, Portugal (Lima, 2009). As demais crianças do estudo, brasileiras, estavam internadas em hospitais públicos: (a) Hospital Estadual Infantil Nossa Senhora da Glória (HEINSG) (Caprini, 2014; Hostert, 2010; Motta, 2007) e Hospital Estadual Infantil e Maternidade Alzir Bernardino Alves (HEIMABA) (Moraes, 2007), no Espírito Santo; e (b) Hospital das Clínicas da Faculdade de Medicina de Botucatu da Universidade Estadual Paulista Júlio de Mesquita Filho (Unesp) (Carnier, 2010), em São Paulo. Todos os hospitais ofereciam aos pacientes pediátricos espaços destinados à recreação, alguns mais estruturados com oferta regular de atividades lúdicas e pedagógicas, outros com menor sistematização.

Os dados analisados foram obtidos a partir da aplicação do AEH - que avalia as estratégias de enfrentamento da hospitalização, em crianças com 6 a 12 anos (Motta \& Enumo, 2002; 2010). Contém 20 pranchas com desenhos que representam os comportamentos de coping: brincar, assistir televisão, cantar, ouvir música, rezar, estudar, conversar, ler gibi, tomar remédio, buscar informações, chorar, sentir raiva, sentir-se triste, desanimar, sentir medo, pensar em fugir, chantagem, esconder, sentir culpa e pensar em milagre. A cada prancha, a criança deve informar, em uma escala tipo Likert, o quanto tem apresentado o comportamento referido para enfrentar a hospitalização: nunca (0) a sempre (4). A partir de inquérito sobre as justificativas das frequências dos comportamentos, O AEH possibilita a análise de estratégias de enfrentamento de acordo com o modelo de Skinner et al. (2003). Entretanto,

334 para este estudo, foram consideradas somente as frequências comportamentais reportadas pelas crianças.

Para o processamento e análise dos dados deste estudo, foram consideradas as variáveis sexo e idade, além do motivo da hospitalização (câncer, cirurgia eletiva e doenças gerais), os quais foram analisados por meio de estatística descritiva e inferencial. Os comportamentos de coping foram apresentados por meio de médias e desvios-padrão. Além disso, as diferenças destes quanto às condições para a hospitalização e ao sexo foram analisadas, respectivamente, por Análise de Variância (Anova) e teste de $t$ para amostras independentes. Análises de correlação também foram empregadas para os comportamentos.

Os estudos analisados consideraram os preceitos éticos para pesquisas com seres humanos, com submissão dos projetos ao parecer dos Comitês de Ética em Pesquisa da Universidade Federal do Espírito Santo (Processo no 217/09), do Hospital Infantil Nossa Senhora da Glória (Processos $n^{\circ}$ 61/2009 e nº1/2013), da Universidade Estadual Paulista Júlio de Mesquita Filho (Processo n 1596/ 46/01/08) e do Instituto Português de Onco-logia de Lisboa Francisco Gentil (Processo n GIC/566), e com início de coleta de dados somente após a sua aprovação, a assinatura do Termo de Consentimento Livre Esclarecido, e do Termo de Assentimento, pela criança.

\section{Resultados}

Os comportamentos de coping mais frequentes no relato das crianças foram tomar remédio, conversar, assistir televisão, rezar e brincar. Em contrapartida, os comportamentos menos frequentes foram sentir culpa, pensar em fugir e se esconder (Tabela 1).

Pelo teste de $t$ para amostras independentes, foi possível encontrar diferenças entre os sexos, com meninas relatando sentir medo mais frequentemente do que os meninos $(p=0,001)$, bem como tristeza $(p=0,015)$. O choro teve maior frequência entre meninas $(p=0,028)$. Apesar disso, as meninas relataram cantar com maior frequência $(p=0,043)$. 
Tabela 1

Médias (M) e Desvios-Padrão (DP) para os comportamentos de coping de crianças hospitalizadas, considerando a amostra geral e o sexo ( $N$ = 148)

\begin{tabular}{|c|c|c|c|c|c|c|c|}
\hline \multirow{2}{*}{ Comportamentos } & \multicolumn{2}{|c|}{ Geral $(N=148)$} & \multicolumn{2}{|c|}{ Feminino $(n=61)$} & \multicolumn{2}{|c|}{ Masculino $(n=87)$} & \multirow{2}{*}{$t^{*}$} \\
\hline & $M$ & $D P$ & $M$ & $D P$ & $M$ & $D P$ & \\
\hline Idade & 9,50 & $( \pm 1,94)$ & 9,72 & $( \pm 1,76)$ & 9,34 & $( \pm 1,77)$ & $-1,16$ \\
\hline Tomar remédio & 3,54 & $( \pm 0,88)$ & 3,44 & $( \pm 0,84)$ & 3,60 & $( \pm 0,89)$ & 1,14 \\
\hline Conversar & 2,79 & $( \pm 1,21)$ & 2,85 & $( \pm 1,17)$ & 2,74 & $( \pm 1,24)$ & $-0,50$ \\
\hline Assistir televisão & 2,77 & $( \pm 1,39)$ & 2,72 & $( \pm 1,40)$ & 2,80 & $( \pm 1,37)$ & 0,35 \\
\hline Rezar & 2,32 & $( \pm 1,65)$ & 2,55 & $( \pm 1,66)$ & 2,14 & $( \pm 1,61)$ & $-1,49$ \\
\hline Brincar & 2,19 & $( \pm 1,45)$ & 2,16 & $( \pm 1,47)$ & 2,20 & $( \pm 1,44)$ & 0,18 \\
\hline Estudar & 1,82 & $( \pm 1,65)$ & 1,68 & $( \pm 1,68)$ & 1,90 & $( \pm 1,61)$ & 0,80 \\
\hline Ler & 1,72 & $( \pm 1,41)$ & 1,95 & $( \pm 1,38)$ & 1,56 & $( \pm 1,41)$ & $-1,66$ \\
\hline Ouvir música & 1,55 & $( \pm 1,50)$ & 1,65 & $( \pm 1,54)$ & 1,48 & $( \pm 1,46)$ & $-0,69$ \\
\hline Sentir medo & 1,47 & $( \pm 1,41)$ & 1,90 & $( \pm 1,41)$ & 1,16 & $( \pm 1,32)$ & $-3,25^{*}$ \\
\hline Buscar informação & 1,36 & $( \pm 1,45)$ & 1,32 & $( \pm 1,48)$ & 1,39 & $( \pm 1,43)$ & 0,26 \\
\hline Sentir tristeza & 1,34 & $( \pm 1,24)$ & 1,63 & $( \pm 1,19)$ & 1,13 & $( \pm 1,23)$ & $-2,47^{*}$ \\
\hline Pensar em milagre & 1,27 & $( \pm 1,51)$ & 1,26 & $( \pm 1,51)$ & 1,27 & $( \pm 1,50)$ & 0,05 \\
\hline Chorar & 1,22 & $( \pm 1,20)$ & 1,47 & $( \pm 1,25)$ & 1,03 & $( \pm 1,13)$ & $-2,22^{*}$ \\
\hline Ficar desanimado & 1,17 & $( \pm 1,21)$ & 1,34 & $( \pm 1,20)$ & 1,04 & $( \pm 1,19)$ & $-1,48$ \\
\hline Cantar & 1,17 & $( \pm 1,38)$ & 1,44 & $( \pm 1,47)$ & 0,97 & $( \pm 1,27)$ & $-2,05^{*}$ \\
\hline Sentir raiva & 1,09 & $( \pm 1,13)$ & 1,24 & $( \pm 1,17)$ & 0,98 & $( \pm 1,08)$ & $-1,37$ \\
\hline Chantagear & 0,72 & $( \pm 1,10)$ & 0,62 & $( \pm 1,05)$ & 0,78 & $( \pm 1,13)$ & 0,86 \\
\hline Sentir culpa & 0,65 & $( \pm 1,08)$ & 0,81 & $( \pm 1,25)$ & 0,52 & $( \pm 0,92)$ & $-1,62$ \\
\hline Pensar em fugir & 0,44 & $( \pm 0,97)$ & 0,44 & $( \pm 0,88)$ & 0,43 & $( \pm 1,02)$ & $-0,08$ \\
\hline Esconder & 0,24 & $( \pm 0,58)$ & 0,27 & $( \pm 0,60)$ & 0,21 & $( \pm 0,55)$ & $-0,62$ \\
\hline
\end{tabular}

Nota: *Valores de $p \leq 0,05$ e significativos pelo teste de $t$ para amostras independentes.

Alguns comportamentos de coping apresentaram correlações significativas entre si (Tabela 2). Serão destacadas as correlações com os comportamentos que apresentaram maior frequência apontada entre as crianças: tomar remédio, conversar, assistir televisão, rezar e brincar.

O comportamento tomar remédio correlacionou-se negativamente com culpar, de modo que aqueles que se culpavam menos pela doença relataram tomar a medicação com mais frequência $(r=-0.18)$. Por sua vez, aqueles que conversavam mais ouviam mais música $(r=0,17)$ e, também, tomavam mais remédio $(r=0,19)$.

O comportamento de assistir televisão correlacionou-se positivamente com estudar $(r=0,36)$, ler $(r=0,22)$ e rezar $(r=0,21)$. Por sua vez, o comportamento rezar correlacionou-se positivamente com estudar $(r=0,25)$, conversar $(r=0,21)$, ouvir música $(r=0,17)$, ler $(r=0,32)$ e buscar informações $(r=0,20)$. Destacam-se, também, rezar e sentir tristeza, que se correlacionaram positivamente $(r=0,17)$, ou seja, as crianças que se sentiam tristes mais frequentemente rezavam mais; e a alta frequência do comportamento de rezar foi igualmente acompanhada de frequência elevada de comportamentos voltados à distração, como estudar e conversar.

Houve correlação significativa entre o brincar e os comportamentos cantar $(r=0,20)$, estudar $(r=0,23)$ e ouvir música $(r=0,16)$. Além disso, houve correlação negativa entre o brincar e os comportamentos de chorar $(r=-0,17)$, sentir raiva $(r=-0,18)$ e se esconder $(r=-0,17)$. Isso indica que as crianças que relataram brincar mais frequentemente cantavam mais, estudavam mais, ouviam mais música no hospital, choravam menos, sentiam raiva menos frequentemente, faziam menos tentativas de se esconder, e vice-versa.

Pelo teste de correlação de Pearson, a idade apresentou correlação significativa apenas com o comportamento de chantagear $(p=0,041)$. A correlação negativa indica que, com o aumento da idade, houve diminuição na frequência relatada para os episódios de chantagem e vice-versa. 
Considerando as condições para a hospitalização (pacientes com câncer, com doenças em geral e em tratamento pré-cirúrgico), houve diferenças quanto aos comportamentos de coping, a partir do teste Anova. Não houve diferença entre os grupos quanto à idade.

O teste post hoc Scheffé comparou os resultados de cada grupo. Os pacientes pré-cirúrgicos relataram rezar mais, segundo o $\mathrm{AEH}$. Ao mesmo tempo, relataram desanimar em menor frequência.
Por outro lado, as crianças com câncer foram as que menos rezavam e mais ficavam desanimadas (Tabela 3).

Os comportamentos de brincar e estudar, por sua vez, foram mais frequentes, respectivamente, entre as crianças com câncer e em hospitalização pré-cirúrgica. As crianças com doenças em geral, num outro polo, apresentaram frequências mais baixas, ou seja, brincavam e estudavam menos.

Tabela 2

Correlações entre os comportamentos de coping em crianças hospitalizadas, aferidos pela Avaliação das Estratégias de Enfrentamento da Hospitalização $(N=148)$

\begin{tabular}{|c|c|c|c|c|c|}
\hline Comportamentos & Tomar remédio & Conversar & Assistir televisão & Rezar & Brincar \\
\hline Cantar e dançar & & & & & 0,20 \\
\hline Rezar & & & 0,36 & & \\
\hline Estudar & & & 0,21 & 0,25 & 0,23 \\
\hline Conversar & & & & 0,21 & \\
\hline Ouvir música & & 0,17 & & 0,17 & 0,16 \\
\hline Ler & & & 0,22 & 0,32 & \\
\hline Tomar remédio & & 0,19 & & & \\
\hline Buscar informações & & & & 0,22 & \\
\hline Chorar & & & & & $-0,17$ \\
\hline Sentir raiva & & & & & $-0,18$ \\
\hline Esconder & & & & & $-0,17$ \\
\hline Sentir tristeza & & & & 0,17 & \\
\hline \multicolumn{6}{|l|}{ Desanimar } \\
\hline \multicolumn{6}{|l|}{ Chantagear } \\
\hline \multicolumn{6}{|l|}{ Pensar em fugir } \\
\hline Sentir culpa & $-0,18$ & & & & \\
\hline \multicolumn{6}{|l|}{ Medo } \\
\hline Pensar em milagre & & & & 0,24 & \\
\hline
\end{tabular}

Nota: Correlações significativas pelo teste de correlação de Pearson, com $p \leq 0,05$.

Tabela 3

Diferença entre médias, de acordo com o motivo da hospitalização, para os comportamentos de coping $(N=148)$

\begin{tabular}{|c|c|c|c|c|c|c|c|}
\hline & \multirow{2}{*}{ Comportamentos } & \multicolumn{2}{|c|}{ Pré-cirurgia $n=59$} & \multicolumn{2}{|c|}{ Câncer $n=61$} & \multicolumn{2}{|c|}{ Enfermaria $n=28$} \\
\hline & & $M$ & $D P$ & $M$ & $D P$ & $M$ & $D P$ \\
\hline \multirow{7}{*}{ 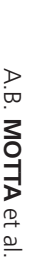 } & Rezar & 2,77 & $\left( \pm 1,48^{\mathrm{a}}\right)$ & 1,93 & $\left( \pm 1,66^{\mathrm{a}}\right)$ & 2,17 & $\left( \pm 1,74^{\mathrm{a}}\right)$ \\
\hline & Ficar desanimado & 0,88 & $\left( \pm 1,11^{\mathrm{a}}\right)$ & 1,47 & $\left( \pm 1,19^{\mathrm{a}}\right)$ & 1,10 & $\left( \pm 1,31^{\text {a }}\right)$ \\
\hline & Brincar & 2,72 & $\left( \pm 1,38^{\mathrm{a}}\right)$ & 2,13 & $\left( \pm 1,25^{a}\right)$ & 1,17 & $\left( \pm 1,46^{b}\right)$ \\
\hline & Estudar & 2,10 & $\left( \pm 1,65^{a}\right)$ & 2,00 & $\left( \pm 1,52^{\mathrm{a}}\right)$ & 0,82 & $\left( \pm 1,54^{b}\right)$ \\
\hline & Ver televisão & 3,20 & $\left( \pm 1,12^{\mathrm{a}}\right)$ & 2,65 & $\left( \pm 1,21^{\mathrm{ab}}\right)$ & 2,10 & $\left( \pm 1,87^{\mathbf{b}}\right)$ \\
\hline & Ouvir música & 1,98 & $\left( \pm 1,53^{\mathrm{a}}\right)$ & 1,45 & $\left( \pm 1,38^{\mathrm{ab}}\right)$ & 0,85 & $\left( \pm 1,40^{b}\right)$ \\
\hline & Cantar & 1,49 & $\left( \pm 1,52^{\mathrm{a}}\right)$ & 1,04 & $\left( \pm 1,20^{\mathrm{ab}}\right)$ & 0,75 & $\left( \pm 1,29^{b}\right)$ \\
\hline & Esconder & 0,11 & $\left( \pm 0,37^{\mathrm{a}}\right)$ & 0,45 & $\left( \pm 0,76^{b}\right)$ & 0,03 & $\left( \pm 0,18^{a}\right)$ \\
\hline & Tomar remédio & 3,55 & $\left( \pm 0,95^{\mathrm{ab}}\right)$ & 3,34 & $\left( \pm 0,91^{\mathrm{a}}\right)$ & 3,92 & $\left( \pm 0,37^{b}\right)$ \\
\hline
\end{tabular}

336 Nota: Médias que não compartilham a mesma letra são significativamente diferentes pelo teste Anova e post hoc Sheffé, com $p \leq 0,05$. 
As crianças com doenças em geral também relataram ver televisão, ouvir música e cantar em menor frequência, em comparação com as crianças sob cuidados pré-cirúrgicos. Já as crianças com câncer não diferiram desses grupos e apresentaram escores intermediários.

Considerando o comportamento de esconder, as crianças com câncer apresentaram escores médios mais altos $(M=0,45 ; \pm 0,76)$, exibindo tal comportamento mais vezes. Por sua vez, as crianças com doenças em geral $(M=0,03 ; \pm 0,18)$ e sob cuidados pré-cirúrgicos $(M=0,11 ; \pm 0,37)$ não diferiram entre si, com escores médios mais baixos.

As crianças das enfermarias relataram, com maior frequência, tomar medicamentos $(M=3,92$; $\pm 0,37$ ), diferindo das crianças com câncer, com médias menos expressivas $(M=3,34 ; \pm 0,91)$. As crianças sob cuidados pré-cirúrgicos compuseram um grupo intermediário para esse critério.

\section{Discussão}

Este estudo descreveu e analisou os comportamentos de coping de crianças frente à hospitalização. Os dados obtidos permitem afirmar que: (a) há correlações entre os comportamentos de coping mais referidos por crianças hospitalizadas; e (b) tais comportamentos se diferenciaram quanto ao sexo e tipo de hospitalização (câncer, cirurgia eletiva e doenças em geral), mas não entre as idades. Com base na Teoria Motivacional do Coping (Skinner et al., 2003; Skinner \& Wellborn, 1994), considera-se a limitação de uma investigação centrada somente nos comportamentos situados na base da estrutura hierárquica do coping (instâncias de coping). Portanto, as análises da relação comportamentos e famílias de coping serão exploratórias.

A maioria dos comportamentos relatados neste estudo refere-se àqueles com maiores chances de um desfecho positivo no processo adaptativo, tais como: tomar remédio, conversar, rezar, assistir televisão e brincar. A depender de sua funcionalidade, esses comportamentos podem se relacionar às famílias de coping de resolução de problemas, distração e busca de suporte, consistente com o fato de que essas famílias, juntamente com a família de coping de esquiva, são as mais comumente encontradas em estudos que avaliam o coping de crianças e adolescentes (Zimmer-Gembeck \& Skinner, 2011). Estudos empíricos com o AEH mostraram que o comportamento de tomar remédio pode vincular-se às estratégias de ação instrumental, com a função de Resolução do problema (Lima, 2009; Motta, 2007). Conversar e rezar, em sua maioria, têm sido justificados pela busca de conforto ou aju$\mathrm{da}$, atendendo à funcionalidade da família Busca de suporte (Motta, 2007). Assistir televisão e brincar são comportamentos típicos de distração (Lima, 2009; Motta \& Enumo, 2010), embora algumas crianças justifiquem o brincar pela regulação emocional (Motta, 2007). Como estratégia de distração, tais comportamentos podem responder pela função da família de coping de Acomodação.

De outro lado, os comportamentos menos frequentes foram aqueles com possível impacto negativo em longo prazo para a adaptação, como sentir culpa, pensar em fugir e se esconder. Nesse ponto, os dados não convergiram com os achados sobre coping, que incluem a Esquiva como estratégia comum no repertório de crianças e adolescentes (Zimmer-Gembeck \& Skinner, 2011), uma vez que os comportamentos de pensar em fugir e se esconder, tipicamente classificados como Esquiva, estiveram entre os menos referidos.

De modo geral, os dados mostram um cenário favorável ao ajustamento psicológico das crianças deste estudo, o que foi confirmado pela análise de correlação entre os comportamentos mais referidos. Assim, a correlação negativa entre tomar remédio e sentir culpa pode ser explicada pelo fato de que ao tomar remédio, a criança emprega uma ação instrumental para a Resolução do problema, que atende sua necessidade de competência (Skinner \& Wellborn, 1994), possivelmente ameaçada pela presença de sentimentos de culpa. Verificou-se também que o comportamento de brincar apresentou correlação positiva com os comportamentos de cantar, estudar e ouvir música. Tais comportamentos podem ter a função de distração; entretanto, a correlação negativa entre brincar e chorar e sentir raiva pode indicar, também, uma função de 
regulação da emoção, com o brincar funcionando para acalmar a criança e diminuir reações de lamentação e irritação (Skinner et al., 2003).

Confirma-se, ainda, a variabilidade de estratégias que as crianças utilizam para enfrentar estressores relacionados à doença e, neste caso, à hospitalização. Se a equipe de saúde conhece esse repertório de comportamentos e estratégias, pode ajudar a criança a utilizá-lo de uma forma positiva (Marsac et al., 2011). Um exemplo seria considerar o brincar, comumente proposto pelo profissional de saúde como técnica de distração comportamental (Duff, 2003), como recurso eficaz a ser empregado intencionalmente pela própria criança em idade escolar, de forma combinada com outras estratégias, tais como foco no positivo e regulação emocional. Ao brincar, a criança pode atribuir outros significados a sua experiência de hospitalização: se a ela é permitida a escolha sobre qual atividade lúdica jogar, devolve-se parte da autonomia; se ao final de um jogo consegue completar a tarefa e sente-se feliz por isso, permite-se a experiência de competência. Isso mostra o quanto as estratégias vão se tornando mais sofisticadas com o avanço de habilidades cognitivas e da autoconfiança, típicas da criança em idade escolar (Zimmer-Gembeck \& Skinner, 2011).

Os dados revelaram ainda uma correlação negativa entre a idade e o comportamento de fazer chantagem. Assim, com o aumento da idade, houve diminuição na frequência relatada para os episódios de chantagem e vice-versa. Sobre essa questão, pode-se considerar que a representação do comportamento por meio de um desenho em que uma criança chora para obter o que deseja de sua mãe tenha influenciado a resposta das crianças. De fato, o choro com esta função pode indicar formas precoces utilizadas por crianças para estabelecer acordos com os adultos. No presente estudo, as crianças tinham em média 9,5 anos e, portanto, apresentavam habilidades cognitivas mais complexas, que Ihes permitiriam empregar outras formas de comunicação, diferentes de chorar. Esse dado pode representar um exemplo de mudanças no coping graduadas pela idade (Skinner \& Zimmer-Gembeck, 338 2009), como sugeriu Katz, Kellerman e Siegel (1980) ao observar que crianças mais novas exteriorizavam suas emoções de forma mais explícita, chorando, gritando e esperneando, enquanto as mais velhas utilizavam formas mais interiorizadas, mudanças na expressão facial e rigidez muscular para negociar. Ao fazer isso, o comportamento seria relacionado a fazer acordos, podendo ter a função de Negociação, família de coping com adaptação positiva (Zimmer-Gembeck \& Skinner, 2011).

A análise de comportamentos de coping em função do sexo evidenciou diferenças significativas, com meninas referindo mais frequentemente reações emocionais de medo, tristeza e choro. Ao avaliar o enfrentamento da dor crônica, Lynch et al. (2007) encontraram nas meninas uma frequência maior de busca por suporte e de expressão de sentimentos, enquanto que, nos meninos, destacaram-se relatos de envolvimento em atividades prazerosas para não pensar na dor, demonstrando que tais diferenças surgem precocemente no desenvolvimento. De fato, o estudo de Vierhaus et al. (2011) mostrou que a percepção e a experiência de dor são mais referidas por meninas do que por meninos.

Em se tratando de diferenças no comportamento de coping em função do tipo de hospitalização, verificou-se uma frequência maior dos comportamentos de coping de brincar e estudar entre as crianças hospitalizadas com câncer e aquelas que seriam submetidas a procedimentos cirúrgicos eletivos, do que entre as crianças hospitalizadas por condições de doenças em geral. A análise desses dados deve considerar aspectos do ambiente hospitalar, especialmente, recursos humanos e materiais que favoreçam o engajamento da criança em atividades lúdicas e de estudo. Nessa subamostra, as crianças com doenças em geral estavam internadas em um hospital infantil da rede pública e, na ocasião da coleta dos dados, não havia entre suas ações a oferta regular de atividades lúdicas e pedagógicas (Moraes, 2007). No caso do câncer, a maioria das crianças tinha acesso a visitas de recreadores, que promoviam e mediavam atividades lúdicas, e contavam com uma Classe Hospitalar para o acompanhamento pedagógico (Hostert, 2010; Motta, 2007). O mesmo ocorreu com as crianças hospi- 
talizadas para procedimentos cirúrgicos eletivos, uma vez que o hospital dispunha de ambientes adequados à recreação, como um parquinho e uma brinquedoteca, além da Classe Hospitalar e de atividades recreativas na enfermaria de pediatria (Carnier, 2010). Assim, recursos e estrutura do ambiente parecem ter contribuído para a homogeneidade do grupo de crianças com câncer e crianças em internação para procedimento cirúrgico eletivo nesses comportamentos.

Apesar das crianças com câncer estarem engajadas em atividades lúdicas, observam-se relatos de desânimo em maior frequência nesse grupo. Entre o grupo de crianças com câncer, a hospitalização ocorreu por diversos fatores, como o diagnóstico da doença, a administração de medicação, a intercorrência do tratamento e o diagnóstico de recidiva, associando a essa condição uma variedade de estressores relacionados ao câncer. Tais estressores podem levar a avaliações diferenciadas sobre sua controlabilidade e, por consequência, eliciar comportamentos e estratégias de enfrentamento diferentes. Assim, embora o brincar, funcionando como uma estratégia de distração comportamental, possa contribuir para a adaptação da criança (Compas et al., 2014), é importante estar atento às reações negativas que podem acompanhar eventos particulares na internação, os quais podem ser percebidos como maior ameaça, levando ao desânimo no enfrentamento da hospitalização. De acordo com Skinner et al. (2003), a reação de desânimo estaria relacionada às famílias Desamparo e Submissão, significando que a hospitalização está sendo percebida como uma ameaça ao self, no âmbito de suas necessidades de competência e autonomia, respectivamente. Deve, portanto, ser foco de atenção para a proposição de intervenções ajustadas às necessidades da criança.

Nesse ponto, as crianças pré-cirúrgicas se diferenciaram das crianças com câncer, já que, além de brincar, essas crianças também rezavam mais e ficavam menos desanimadas. Diante do encontro com o desconhecido, esperam-se reações de ansiedade, as quais são manejadas a partir de estratégias de enfrentamento variadas, entre elas o uso da religiosidade (Fighera \& Viero, 2005). Assim, o suporte social é dirigido a Deus, como possibilidade de conforto e segurança, podendo explicar a alta frequência do comportamento de rezar entre as crianças em preparação para cirurgia. O uso da religiosidade poderia atuar, também, no nível da regulação do estado emocional da criança, uma vez que motivadas pela esperança obtida na oração, poderiam sentir menos desânimo e medo. Todavia, novos estudos são necessários para avaliar com maior precisão a relação entre rezar e desânimo no hospital.

Outro ponto de análise entre os grupos foi a diferenciação das crianças com doenças em geral e câncer em relação ao comportamento de tomar remédio. É possível que, ao avaliar o contexto do enfrentamento (Peterson, 1989), a criança do grupo de crianças internadas com doenças diversas compare a ingestão de medicamentos atual com a sua experiência pregressa (quando não estava doente), levando a uma percepção superdimensionada desse comportamento. De outro lado, no câncer, que se caracteriza como uma doença crônica, com necessidade de hospitalizações recorrentes, o comportamento de tomar remédio passa a ser mais familiar à criança, sendo esperada a sua ocorrência, mesmo quando está em tratamento ambulatorial. Isso poderia levar a avaliação da ingestão de medicamentos como sendo baixa, especialmente, quando comparadas ao período inicial de diagnóstico e tratamento.

Em relação à subamostra de crianças com câncer, é importante destacar sua característica multicultural. Crianças brasileiras e portuguesas em tratamento de câncer apresentam semelhanças em seus comportamentos de coping na hospitalização (Lima, 2009; Lima et al., 2014), o que permitiu a composição de um único grupo de crianças com câncer. Somente quando se analisa os comportamentos menos referidos, é possível encontrar, entre as crianças portuguesas, frequências menores dos comportamentos de se esconder, pensar em fugir e cantar e dançar. Estes, por sua vez, já haviam sido menos referidos entre as crianças brasileiras, o que dificulta conclusões a respeito de diferenças justificadas por variações culturais. Estudos subsequentes com foco em análises transculturais poderão esclarecer tais achados e se mostram relevantes 
quando se utiliza um instrumento que pretende buscar evidências de validade, como é o caso do AEH (Garioli, 2013).

Limitações do presente estudo devem ser consideradas, especialmente, para a proposição de pesquisas subsequentes. A começar pelo tamanho da amostra que, apesar de ampliada com a integração dos dados, é reduzido quando se agrupam as crianças por diagnóstico, e não há uma distribuição equivalente do número de crianças em cada grupo. Além disso, os dados foram coletados em diferentes hospitais, o que pode levar a uma consideração de que os resultados do estudo sejam, em parte, função de diferenças na oferta de recursos nos locais de pesquisa, tal como foi discutido na explicação da maior ocorrência de brincar e estudar em hospitais que oferecem meios para a recreação em brinquedotecas e classes hospitalares.

Por fim, o presente estudo é o primeiro a agregar dados sobre o enfrentamento de crianças hospitalizadas, por meio do AEH, contribuindo para a compreensão sobre as relações entre sexo, idade, motivo da hospitalização e comportamentos de coping. Dada a relevância do coping para o ajustamento da criança, estudos futuros poderiam incluir medidas após a hospitalização verificando diferenças quando esta se dá por motivos agudos, em que não há perspectiva de novo encontro com o estressor e, por motivos crônicos, em que a recorrência do evento é iminente. Tais esclarecimentos poderiam guiar propostas de intervenção que se diferenciam no ambiente hospitalar, para atender as necessidades da criança.

\section{Referências}

Barros, L. (2003). As consequências psicológicas da hospitalização infantil: Prevenção e controlo. In L. Barros. Psicologia pediátrica: perspectiva desenvolvimentista (2 ${ }^{a}$ ed., pp.69-92). Lisboa: Climepsi.

Caprini, F. R. (2014). Do diagnóstico ao tratamento: compreendendo o processo de enfrentamento da criança com câncer (Dissertação de mestrado não-publicada). Universidade Federal do Espírito Santo, Vitória.

Carnier, L. E. (2010). Stress e coping em crianças hospitalizadas em situação pré-cirúrgica e stress do acompanhante: estabelecendo relações (Dissertação de mestrado não-publicada). Universidade Estadual
Compas, B. E., \& Boyer, M. C. (2001). Coping and attention: Implications for child health and pediatric conditions. Developmental and Behavioral Pediatrics, 22(5), 323-333. http://dx.doi.org/10.1097/00004703-2 00110000-00007

Compas, B. E., Connor-Smith, J. K., Saltzman, H., Thomsen, A. H., \& Wadsworth, M. E. (2001). Coping with stress during childhood an adolescence: Problems, progress, and potential in theory and research. Psychological Bulletin, 127(1), 87-127. http:// dx.doi.org/10.1037/0033-2909.127.1.87

Compas, B. E., Desjardins, L., Vannatta, K., Young-Saleme, T., Rodriguez, E. M., Dunn, ... Gerhardt, C.A. (2014). Children and adolescents coping with cancer: Selfand parents reports of coping and anxiety/depression. Health Psychology, 33(8), 853-861. http://dx.doi.org/ 10.1037/hea0000083

Compas, B. E., Jaser, S. S., Dunn, M. J., \& Rodriguez, E. M. (2012). Coping with chronic illness in childhood and adolescence. Annual Reviews Clinical Psychology, 27(8), 455-480. http://dx.doi.org/10.1146/annurevclinpsy-032511-143108

Duff, A. J. A. (2003). Incorporating psychological approaches into routine paediatric venepuncture. Archives of Disease in Childhood, 88(10), 931-937. http://dx.doi.org/10.1136/adc.88.10.931

Fighera, J., \& Viero, E. V. (2005). Vivências do paciente com relação ao procedimento cirúrgico: Fantasias e sentimentos mais presentes. Revista Brasileira de Psicologia Hospitalar, 8(2), 51-63.

Franck, L. S., Wray J., Gay C., Dearmun A. K., Lee K., \& Cooper B. A. (2014). Predictors of parent posttraumatic stress symptoms after child hospitalization on general pediatric wards: A prospective cohort study. International Journal of Nursing Studies, 52(1), 10-21. http://dx.doi.org/10.1016/j.ijnurstu.2014.06.011

Garioli, D. S. (2011). O impacto da dor nas funções executivas e sua relação com as estratégias de enfrentamento em crianças com Anemia Falciforme (Dissertação de mestrado não-publicada). Universidade Federal do Espírito Santo, Vitória.

Garioli, D. S. (2013). Avaliação das Estratégias de Enfrentamento da Hospitalização (AEH 2.0): processo de adaptação de uma escala (Projeto de tese de doutorado não-publicada). Universidade Federal do Espírito Santo, Vitória.

Haiat, H., Bar-Mor, G., \& Shochat, M. (2003). The world of the child: A world of play even in the hospital. International Pediatric Nursing, 18(3), 209-214.

Hostert, P. C. C. P. (2010). Estratégias de enfrentamento e problemas comportamentais em crianças com câncer, na classe hospitalar (Dissertação de mestrado não-publicada). Universidade Federal do Espírito Santo, Vitória.

Katz, E. R., Kellerman, J., \& Siegel, S. E. (1980). Behavioral distress in children with cancer undergoing medical 
procedures: Developmental considerations. Journal of Consulting and Clinical Psychology, 48(3), 356-365. http://dx.doi.org/10.1037/0022-006X.48.3.356

Lima, A. S. S. (2009). Avaliação de estratégias de coping em crianças com cancro: estudo exploratório (Dissertação de mestrado não-publicada). Universidade de Lisboa.

Lima, A. S., Barros, M. L. T. Q., \& Enumo, S. R. F. (2014). Análise de dois instrumentos de coping aplicados em crianças portuguesas hospitalizadas. Estudos de Psicologia, 31(4), 559-571. http://dx.doi.org/10.1590/ 0103-166X2014000400010

Lynch, A. M., Kashikar-Zuck, S., Goldschneider, K. R., \& Jones, B. A. (2007). Sex and age diferences in coping styles among children with chronic pain. Journal of Pain and Symptom Management, 33(2), 208-216. http://dx.doi.org/10.1016/j.jpainsymman.2006.07. 014

Marsac, M. L., Donlon, K. A., Winston, F. K., \& KassamAdams, N. (2011). Child coping, parent coping assistance, and post-traumatic stress following paediatric physical injury. Child: Care, health and development, 39(2), 171-177. http://dx.doi.org/ 10.1111/j.1365-2214.2011.01328.x

Moraes, E. O. (2007). Avaliação do enfrentamento da hospitalização em crianças por meio de instrumentos informatizados (Dissertação de mestrado não-publicada). Universidade Federal de Espírito Santo, Vitória.

Moraes, E. O., \& Enumo, S. R. F. (2008). Estratégias de enfrentamento da hospitalização em crianças avaliadas por instrumento informatizado. Psico USF, 13(2), 221-231.

Motta, A. B. (2007). Brincando no hospital: uma proposta de intervenção psicológica para crianças internadas com câncer (Tese de doutorado não-publicada). Universidade Federal do Espírito Santo, Vitória.

Motta, A. B., \& Enumo, S. R. F. (2002). Brincar no hospital: avaliação do enfrentamento da hospitalização. Psicologia: Saúde \& Doença, 3(1), 23-41.

Motta, A. B., \& Enumo, S. R. F. (2010). Intervenção psicológica lúdica para o enfrentamento da hospitalização em crianças com câncer. Psicologia: Teoria e Pesquisa, 26(3), 445-454. http://dx.doi.org/10.1590/S 0102-37722010000300007

Oliveira, C. G. T. (2013). Enfrentamento da dor na anemia falciforme em crianças: avaliação e intervenção (Tese de doutorado não-publicada). Universidade Federal do Espírito Santo, Vitória.
Peterson, L. (1989). Coping by children undergoing stressful medical procedures: Some conceptual, methodological, and therapeutic issues. Journal of Consulting and Clinical Psychology, 57(3), 380-387.

Ramos, F. P. (2012). Uma proposta de análise do coping no contexto de grupo de mães de bebês prematuros e de baixo peso na Unidade de Terapia Intensiva Neonatal (Tese de doutorado não-publicada). Universidade Federal do Espírito Santo, Vitória.

Rodriguez, E. M., Dunn, M. J., Zuckerman,T., Vannatta, K., Gerhardt, C. A., \& Compas, B. E. (2012). Cancer-related sources of stress for children with cancer and their parents. Journal of Pediatric Psychology, 37(2), 185-197. http://dx.doi.org/10.1093/jpepsy/jsr054

Skinner, E. A., Edge, K., Altman, J., \& Sherwood, H. (2003). Searching for the structure of coping: A review and critique of category systems for classifying ways of coping. Psychological Bulletin, 129, 216-269. http:// dx.doi.org/10.1037/0033-2909.129.2.216

Skinner, E. A., \& Wellborn, J. G. (1994). Coping during childhood and adolescence: A motivational perspective. In D. Featherman, R. M. Lerner, \& M. Perlmutter (Eds.), Life-span development and behavior (pp.91-133). Hillsdale: Erlbaum.

Skinner, E. A., \& Zimmer-Gembeck, M. J. (2009). Challenges to the developmental study of coping. In E. A. Skinner \& M. J. Zimmer-Gembeck (Eds.), Coping and the development of regulation: New directions for child and adolescent development (pp.5-17). San Francisco: Jossey-Bass.

Vierhaus, M., Lohaus, A., \& Schmitz, A. (2011). Sex, gender, coping, and self-efficacy: Mediation of sex diferences in pais perception in children and adolescentes. European Journal of Pain, 15, 621.e1-621.e8. http:// dx.doi.org/10.1016/j.ejpain.2010.11.003

Zimmer-Gembeck, M. J., \& Skinner, E. A. (2009). Coping, developmental influences. In H. Reis, \& S. Sprecher (Eds.), Encyclopedia of human relationships (pp.332335). Newbury Park: Sage.

Zimmer-Gembeck, M. J., \& Skinner, E. A. (2011). The development of coping across childhood and adolescence: An integrative review and critique of research. International Journal of Behavioral Development, 35(1), 1-17. http://dx.doi.org/10.1177/ 0165025410384923

Recebido: dezembro 15, 2014

Aprovado: janeiro 12, 2015 
